\title{
Perfect approximation of functions
}

\section{A.J. van der Poorten}

\begin{abstract}
There are only isolated instances of vectors of functions for which it is possible to obtain an explicit expression for the remainder functions obtained upon approximating by polynomials in the manner described by Mahler in his paper "Perfect systems", Compositio Math. 19 (1968). We display appropriate identities and point to a pattern amongst these which suggests we should not expect convenient generalization to wider classes of functions. Proofs of perfectness do not require laborious computation but are immediate from the identities given.
\end{abstract}

1.

Within the theory of transcendental numbers the approximation of functions by polynomials, and thus in effect by rational functions, is a technique of fundamental importance. The entire theory of exponential polynomials and its applications ranging from study of the Riemann $\zeta$-function to the stability theory of aifferential equations is appropriately viewed as a special case of polynomial approximation.

We briefly describe the theory of approximation of functions developed by Mahler [5] and extended by Coates [2, 3] with a view to displaying the few instances where it is possible to explicitly give the polynomials approximating a vector of functions. The common pattern of these constructions does not appear to have been previously observed upon. Nor is it apparent from these results in isolation that there is little reason to expect convenient generalization. to classes of functions other than those for which exact formulae are already available.

Received 2 February 1971. 
The ideas of Mahler's theory of perfect systems are as follows:

Let $G$ be an open connected subset of the complex plane, and let $\Omega=\Omega: z_{1}, z_{2}, \ldots$ denote an arbitrary sequence of points of $G$. We write

$$
\psi_{0}(z)=1, \quad \psi_{\lambda}(z)=\left(z-z_{1}\right)\left(z-z_{2}\right) \ldots\left(z-z_{\lambda}\right), \lambda=1,2, \ldots .
$$

Then for every non-negative integer $n$, a function $f$, analytic on $G$, has a unique interpolation expansion with respect to $\Omega$ :

$$
f(z)=\sum_{\lambda=0}^{n-1} b_{\lambda} \psi_{\lambda}(z)+s_{n}(z) \psi_{n}(z),
$$

where the $b_{\lambda}$ are constants, and $s_{n}$ is analytic on $G$. If $b_{k} \neq 0$, but $b_{\lambda}=0 \quad(\lambda=0,1, \ldots, k-1)$, we say that the order of $f$ with respect to $\Omega$ is $k$, and we write

$$
w_{\Omega}[f]=k \text {. }
$$

We remark that in the case $\Omega: w, w, \ldots$, where $\Omega$ consists of a single point $w$ repeated, the interpolation series (1) becomes the Taylor expansion at $w$, and the order becomes the order of the zero at $w$. In general, $w_{\Omega}$ is a pseudo-valuation on the ring of functions analytic on $G$.

We shall denote the degree of a polynomial $p$ by

$$
\partial[p]
$$

with the convention that the identically vanishing polynomial has degree -1 .

Let $m$ be a positive integer and denote by $f$ the vector $\left(f_{1}(z), \ldots, f_{m}(z)\right)$ of functions analytic on $G$. Further let $\rho$ denote the vector $(\rho(1), \ldots, \rho(m))$ of non-negative integers with sum $\rho(1)+\ldots+\rho(m)=\sigma$.

Then we may select a vector $p$ of polynomials $\left(p_{1}(z), \ldots, p_{m}(z)\right)$, not all vanishing identically, such that

$$
\partial\left[p_{k}\right] \leq \rho(k)-1 \quad(k=1, \ldots,)
$$


and

$$
w_{\Omega}\left[\sum_{k=1}^{m} p_{k}(z) f_{k}(z)\right] \geq \sigma-1 .
$$

To see this can always be done we need only observe that the requirements (2) impose just $\sigma-1$ linear conditions on the $\sigma$ coefficients of the polynomials of $p$.

It may be that the vector $f$ is such that

(3) $\partial\left[p_{k}\right] \leq \rho(k)-1 \quad(k=1, \ldots, m) \Rightarrow w_{\Omega}\left[\sum_{k=1}^{m} p_{k}(z) f_{k}(z)\right] \leq \sigma-1$.

We then say that $f$ is $\Omega$-normal at $\rho$. This concept is due to Coates [2] who localised the concept of perfectness due to Mahler [5], namely that $f$ is $\Omega$-perfect if it is $\Omega$-normal at $\rho$, for all $\rho$.

Implications of normality and perfectness are given in detail in Coates' papers [2, 3]. In particular, as a criterion, we might note that $f$ is $\Omega$-perfect if and only if the conditions (2) uniquely determine the vector $p$ at each $p$ up to normalisation, or equivalently if necessarily (2) implies $\partial\left[p_{k}\right]=\rho(k)-1(k=1, \ldots, m)$.

There are a variety of applications for conditions which guarantee that functions not vanish too often. Thus in the theory of transcendental numbers, the Siegel method requires the construction of systems of linearly independent forms

$$
r_{h}=\sum_{k=1}^{m} a_{h k} f_{k}(\alpha) \quad(h=1,2, \ldots, m)
$$

in the quantities $f_{1}(\alpha), \ldots, f_{m}(\alpha)$ to be studied. $\Omega$-perfectness of $f$ already implies the possitility of constructing infinitely many such systems for points $\alpha$ of $G / \Omega$. Indeed it is appropriate to assert that in general proofs in the theory of transcendental numbers depend on constructing a non-trivial auxiliary function. Then on the one hand some assumption of algebraic dependence leads the function to vanish often on some sequence; on the other hand known properties of the function then imply it must vanish identically, thus contradicting the assumption. 
2.

For most applications it is only necessary to be able to estimate the coefficients of the polynomials $p_{k}(z)$ satisfying (2). For accurate transcendence measures explicit 'tidy' evaluations are however desirable. It is interesting, though not entirely surprising, that with one general exception described below, the very few instances of perfectness that we know are exactly those in which we are able to give the approximating polynomials $p_{k}(z)$ in a convenient closed form.

Although we are able to give only few explicit instances of perfectness or even of vectors $f \Omega$-normal for an infinite set of $\rho$, Coates [3] shows that these phenomena are by no means unusual. In particular, suppose $f$ is such that the functions $f_{1}(z), \ldots, f_{m}(z)$ are linearly independent over the field of rational functions. If $S$ is a countable subset of $G$ then there exist infinitely many sequences $\Omega$ of points of $G$, so that $f$ is $\Omega$-perfect, and so that for each point of $S$ there is a subsequence of $\Omega$ converging to the point. Moreover if the functions $f_{1}(z), \ldots, f_{m}(z)$ do not vanish simultaneously at any point of $\Omega$, then there are infinitely many $\rho$ at whj.ch $f$ is $\Omega$-nornal.

\section{3.}

The only general explicit non-trivial instance of perfectness is the result contained in a problem of Pólya and Szegö [9; p. 49, Nr. 76] and a lemma of Gelfond and Linnik [4; p. 232]: if $\alpha_{1}, \ldots, \alpha_{m}$ cre distinct real numbers, then the vector $\left(e^{\alpha_{1} z}, \ldots, e^{\alpha_{m} z}\right)$ is $\Omega$-perfect for all sequences $\Omega$ of real numbers. The result is an elementary consequence of Rolle's Theorem. Here we cannot generally give the approximating polynomials in tidy closed form.

In the few further cases we however have attractive integral formulae. Thus consider (Mahler [6]):

$$
F(z)=\sum_{k=1}^{m} \sum_{s=1}^{\rho(k)} a_{k s} z^{s-1} e^{\alpha_{k} z}=\frac{1}{2 \pi i} \int_{C} \prod_{k=1}^{m}\left(\zeta-\alpha_{k}\right)^{-\rho(k)} e^{\zeta z} d \zeta,
$$


where the closed contour $C$ contains the distinct complex numbers $\alpha_{1}, \ldots, \alpha_{m}$.

It is easy to see, by looking outside the contour $C$, that we have

$$
{ }_{F}^{(\lambda-1)}(0)=0 \quad(\lambda=1,2, \ldots, \sigma-1) ; F^{(\sigma-1)}(0)=1 .
$$

Moreover each of the polynomials $\sum_{s=1}^{\rho(k)} a_{k s} z^{s-1}=p_{k}(s)$ attains its exact degree $\rho(k)-1$, the leading coefficient being

$$
\frac{1}{(\rho(k)-1) !} \prod_{h \neq k}\left(\alpha_{k}-\alpha_{h}\right)^{-\rho(h)} \neq 0 \quad(k=1, \ldots, m) \text {. }
$$

It therefore follows that if $\alpha_{1}, \ldots, \alpha_{m}$ are distinct complex numbers then the vector $\left(e^{\alpha_{1} z}, \ldots, e^{\alpha_{m}}\right)$ is perfect with respect to the sequence $\Omega: 0,0,0, \ldots$. The identity (4) provides explicit expressions for the approximating polynomials and permits convenient estimation of the remainder function $F(z)$.

Similarly we have (Coates [2]):

$$
F(z)=\sum_{k=1}^{m} \sum_{s=1}^{\rho(k)} a_{k s} z^{s-1} \alpha_{k}^{z}=\frac{1}{2 \pi i} \int_{C} \prod_{k=1}^{m}\left(\zeta-\alpha_{k}\right)^{-\rho(k)} \zeta^{z} d \zeta
$$

where, as above, the closed contour $C$ contains the distinct non-zero complex numbers $\alpha_{1}, \ldots, \alpha_{m}$.

We see that

$$
F(\mu)=0 \quad(\mu=0,1, \ldots, \sigma-2) ; F(\sigma-1)=1
$$

and each of the polynomials $\sum_{k=1}^{m} a_{k s} z^{s-1}=p_{k}(z)$ attains its exact degree $\rho(k)-1$. Thus if $\alpha_{1}, \ldots, \alpha_{m}$ are distinct non-zero complex nombers, the vector $\left(\alpha_{1}^{z}, \ldots, \alpha_{m}^{z}\right)$ is perfect with respect to the sequence $\Omega: 0,1,2, \ldots$.

Finally in this class of results we have (Mahler [7]): 


$$
\begin{aligned}
F(z) & =\sum_{k=1}^{m} \sum_{s=1}^{\rho(k)} a_{k s} z^{s-1}(1-z)^{\alpha} \\
& =\frac{1}{2 \pi i} \int_{C} \prod_{k=1}^{m} \prod_{s=1}^{\rho(k)}\left(\zeta-\alpha_{k}-s+1\right)^{-1}(1-z)^{\zeta} d \zeta,
\end{aligned}
$$

where the closed contour $C$ contains the distinct numbers $\alpha_{k}+s-1$ $(k=1, \ldots, m ; s=1, \ldots, \rho(k))$. It follows, as in the first example, that if no two of the complex numbers $\alpha_{1}, \ldots, \alpha_{m}$ differ by a rational

integer, then the vector $(1-z)^{\alpha}, \ldots,(1-z)^{\alpha}$ is perfect with respect to the sequence $\Omega: 0,0,0, \ldots$.

It is not difficult to guess the above identities. Thus we have

$$
\sum_{k=1}^{m} b_{k} e^{\alpha_{k} z}=\frac{1}{2 \pi i} \int_{C} \prod_{k=1}^{m}\left(\zeta-\alpha_{k}\right)^{-1} \cdot e^{\zeta z} d \zeta
$$

where the numbers $b_{k}$ are functions of $\alpha_{1}, \ldots, \alpha_{m}$. If we now apply the operator

$$
\prod_{k=1}^{m} \frac{1}{(\rho(k)-1) !}\left(\frac{\partial}{\partial \alpha_{k}}\right)^{\rho(k)-1}
$$

then we immediately obtair Mahler's identity (4).

We apply the above approach to obtain an identity which proves a normality theorem due to Baker [1]. We see immediately that

$$
\sum_{k=1}^{m} \sum_{s=1}^{\rho(k)} b_{k s}(1-z)^{\alpha k s}=\frac{1}{2 \pi i} \int_{C} \prod_{k=1}^{m} \prod_{s=1}^{\rho(k)}\left(\zeta-\alpha_{k s}\right)^{-1}(1-z)^{\zeta} d \zeta,
$$

where of course the closed contour $C$ contains all the points $\alpha_{k s}$, and we note that the numbers $b_{k s}$ are functions of the $\alpha_{k s}$. Now let $\tau(k, i s) \quad(k=1, \ldots, m ; s=1,2, \ldots, p(k))$ be positive integers satisfying

$$
\tau(k, 1) \geq \tau(k, 2) \geq \ldots \geq \tau(k, \rho(k)) \quad(k=1, \ldots, m)
$$

and apply the operator

$$
\prod_{k=1}^{m} \prod_{s=1}^{\rho(k)} \frac{1}{(\tau(k, s)-1) !}\left(\frac{\partial}{\partial \alpha_{k s}}\right)^{\tau(k, s)-1},
$$


finally putting $\alpha_{k s}=\alpha_{k}+s-1(k=1, \ldots, m ; s=1, \ldots, \rho(k))$, these numbers being assumed distinct. Then we obtain an identity of the form

$$
\begin{aligned}
F(z) & =\sum_{k=1}^{m} \sum_{p=1}^{n(k)} \sum_{s=1}^{\rho(k, p)} a_{k p s} z^{s-1}(\log (1-z))^{p-1}(1-z) \alpha_{k} \\
& =\frac{1}{2 \pi i} \int_{C} \prod_{k=1}^{m} \prod_{s=1}^{\rho(k)}\left(\zeta-\alpha_{k}-s+1\right)^{-\tau(k, s)}(1-z)^{\zeta} d \zeta,
\end{aligned}
$$

where

$$
\rho(k, p)=\max \{s: \tau(k, s) \geq p\}
$$

It follows that

$$
\rho(k)=\rho(k, 1) \geq \rho(k, 2) \geq \ldots \geq \rho(k, n(k)) \quad(k=1,2, \ldots, m)
$$

and, again for $k=1,2, \ldots, m$,

$$
\sum_{s=1}^{\rho(k)} \tau(k, s)=\sum_{p=1}^{n(k)} \rho(k, p)=\sigma(k) \text {, }
$$

say. We see, as before, that if $\sum_{k=1}^{m} \sigma(k)=\sigma$,

$$
{ }_{F}^{(\lambda-1)}(0)=0 \quad(\lambda=1,2, \ldots, \sigma-1), F^{(\sigma-1)}(0)=(-1)^{\sigma-1} .
$$

It then follows by observing the degrees attained by the polynomial coefficients, that if no two of the complex numbers $\alpha_{1}, \ldots, \alpha_{m}$ differ by a rational integer the doubly-indexed vector of functions $(\log (1-z))^{p-1}(1-z)^{\alpha_{k}} \quad(k=1, \ldots, m ; p=1, \ldots, n(k))$ is normal with respect to $\Omega: 0,0,0, \ldots$ at all points $\rho(k, p)$ $(k=1, \ldots, m ; p=1, \ldots, n(k))$ satisfying

$$
\rho(k, 1) \geq \rho(k, 2) \geq \ldots \geq \rho(k, n(k)) \quad(k=1, \ldots, m) .
$$

This result of course includes the perfectness theorem for the binomial vector given above, as well as the result due to Mahler [8] that the vector $\left(1, \log (1-z), \ldots,(\log (1-z))^{n-1}\right)$ is normal with respect to $\Omega: 0,0,0, \ldots$ at all points $\rho$ satisfying $\rho(1) \geq \rho(2) \geq \ldots \geq \rho(n)$. 
The results quoted depend upon convenient properties of the functions $e^{\zeta z}, \zeta^{z}$ and $(1-z)^{\zeta}$. Consideration of this observation allows one to produce one further identity:

$$
\sum_{k=1}^{m} \sum_{s=1}^{\rho(k)} a_{k s} z^{s-1} \Gamma\left(z+\alpha_{k}\right)=\frac{1}{2 \pi i} \int_{C} \prod_{k=1}^{m} \prod_{s=1}^{\rho(k)}\left(\zeta-\alpha_{k}-s+1\right)^{-1} \frac{\Gamma(z+\zeta)}{\Gamma(\zeta)} d \zeta
$$

and the consequent, if no two of the complex numbers $\alpha_{1}, \ldots, \alpha_{m}$ differ by a rational integer and none is a non-positive rational integer, the vector $\left(\Gamma\left(z+\alpha_{1}\right), \ldots, \Gamma\left(z+\alpha_{m}\right)\right)$ is perfect with respect to $\Omega: 0,1,2, \ldots$.

There is no reason to expect to obtain similar identities for wider classes of functions.

It would be invaluable to be able to display multidimensional generalizations of the identities given, even though only exponential and logarithmic functions might appear. Indeed, there are at present virtually no results relating the arithmetic properties of logarithms and exponentials. Unfortunately integration over polydisc domains does not appear to provide results of the right form, nor for other domains do the appropriate reproducing kernels appear amenable to manipulation.

It is easy to see that from an alternative viewpoint the normality of a function vector at a parameter point $\rho$ is a matter of the non-vanishing of the determinant of a system of $\sigma$ linear equations in the $\sigma$ coefficients of the approximating polynomials. In exactly the cases described above it is possible to evaluate these determinants in a suitable closed form, the determinants proving to be generalisations of the well known Vandermonde alternant. Starting from the determinantal viewpoint, the normality results of this paper have been applied to obtain generalisation of Turán's Main Theorems on lower bounds for sums of powers $[10,11]$. One may further obtain a number of isolated normality results for vectors of periodic functions. These will be discussed in a subsequent paper [12]. 


\section{References}

[1] A. Baker, "A note on the Padé table", K. Nederl. Akad. Wetensch. Proc. Ser. A 69 (1966), 596-601.

[2] John Coates, "On the algebraic approximation of functions. I, II, III", K. NederZ. Akad. Wetensch. Proc. Ser. A 69 (1966), $421-434,435-448,449-461$.

[3] John Coates, "On the algebraic approximation of functions. IV", $K$. Nederl. Akad. Wetensch. Proc. Ser. A 70 (1967), 205-212.

[4] A.O. Gelfond and Yu.V. Linnik, Elementary methods in analytic number theory (Translated by Amiel Feinstein. Revised and edited by L.J. Mordell. Rand McNally, Chicago, 1965; George Allen and Unwin, London, 1966).

[5] K. Mahler, "Perfect systems", Compositio Math. 19 (1968), 95-166.

[6] Kurt Mahler, "Zur Approximation der Exponentialfunktion und des Logarithmus, I, II", J. reine angew. Math. 166 (1931), 118-136, $137-150$.

[7] Kurt Mahler, "Ein Beweis des Thue-Siegelschen Satzes über die Approximation algebraischer Zahlen für binomische Gleichungen", Math. Ann. 105 (1931), 267-276.

[8] K. Mahler, "On the approximation of logarithms of algebraic numbers" PhiZos. Trans. Roy. Soc. London Ser. A 245 (1953), 371-398.

[9] G. Pólya und G. Szegö, Aufgạben und Lehrsätze aus der Analysis, Zweiter Band, 2te Aufl. (Die Grundlehren der mathematischen Wissenschaften in Einzeldarstellungen, Band 20, Springer-Verlag, Berlin, Göttingen, Heidelberg, 1954).

[10] A.J. van der Poorten, "Generalizations of Turán's main theorems on lower bounds for sums of powers", Bulz. Austral. Math. Soc. 2 (1970), 15-37.

[11] A.J. van der Poorten, "A generalization of Turán's main theorems to binomials and logarithms", Bull. Austral. Math. Soc. 2 (1970), 183-195. 
[12] A.J. van der Poorten, "Generalised Vandermonde determinants", (to appear).

The University of New South Wales, Kensington,

New South Wales. 